\title{
DOI: 10.36622/VSTU.2021.47.26.007
}

\section{УДК 658.27}

\section{СИСТЕМА ПРИНЦИПОВ УПРАВЛЕНИЯ ОСНОВНЫМИ СРЕДСТВАМИ ПРЕДПРИЯТИЯ}

\author{
В.В. Кобзев, М.К. Измайлов \\ Санкт-Петербургский политехнический университет Петра Великого (СПбПУ) \\ Россия, 195251, Санкт-Петербург, ул. Политехническая, д. 29
}

Введение. В статье проведено исследование основных подходов к систематизации принципов управления основными средствами предприятия. Во вступительной части статьи обоснована важность и необходимость формулировки научно обоснованных принципов управления основными средствами. Далее в основной части статьи приведены мнения отдельных авторов относительно видов принципов управления основныли средствами предприятия, на основе чего сделан вывод, что различные авторы приводят в своих работах собственные подходы к классификации принципов управления основными средствами. Обосновано, что ни один из приведенных перечней принципов не является исчерпывающим, в большинстве они носят достаточно общий характер, могут быть использованы в отношении не только основных средств, но практически любого объекта управления на предприятии.

Полученные результаты. В результате исследования сформирована система специальных приниипов управления основными фондами предприятия, которая представлена в виде схемы с подробной характеристикой. Система включает в себя следующие принщипы: 1) принщип комплексного учета институтов и институциональных изменений, связанных с основными средствами; 2) принцип рационализачии трансакиионных издержек, возникающих в прочессе воспроизводства основных средств; 3) приниип рациональной транспарентности процесса управления основными средствами; 4) принци полисубъектности управления основными средствами предприятия; 5) принцип синергизма; 6) принцип нацеленности на долгосрочную максимизацию эффективности и стоимости промышленного предприятия. Предлагаемая система принципов управления основными фондами предприятия позволяет наиболее полно отразить специфику основных средств как объекта управления, а также обобщить и систематизировать принщипы, приведенные в исследуемых литературных источниках.

Заключение. Соблюдение требований предлагаемых принципов позволит обеспечить высокое качество оперативно-производственной деятельности, a также создает условия для совершенствования организационно-экономического обеспечения поддержания рациональной структуры основных производственных средств в прочессе достижения иелей экономической деятельности предприятий и, соответственно, повышение адаптивности к требованиям рынка, стимулирование ожсиления деловой активности. В свою очередь, эффективно сформированная система управления основными фондами позволит обеспечить рост объемов производимой продукции, повышение качества, увеличение уровня производительности труда, сокращение затрат на производство.

\section{Сведения об авторах:}

Кобзев Владимир Васильевич (max78rus@ya.ru), д-р экон. наук, профессор Санкт-Петербургского политехнического университета Петра Великого (СПбПУ)

Измайлов Максим Кириллович (max78rus@ya.ru), ассистент Санкт-Петербургского политехнического университета Петра Великого (СПбПУ)
On authors:

Vladimir V. Kobzev (max78rus@ya.ru), doctor of Economics, Professor of Peter the Great St. Petersburg Polytechnic University Maxim K. Izmailov (max78rus@ ya.ru), assistant at the Institute of Peter the Great St. Petersburg Polytechnic University 
Ключевые слова: основные средства, управление, принципы управления, основныле фонды, эффективность использования, промышленные предприятия, система принципов.

\title{
Для цитирования:
}

Кобзев В.В., Измайлов М.К. Система принципов управления основными средствами // Организатор производства. 2021. Т.29. № 2. C. 67-76. DOI: 10.36622/VSTU.2021.47.26.007.

\section{THE SYSTEM OF PRINCIPLES OF MANAGEMENT OF FIXED ASSETS}

\author{
V. V. Kobzev, M. K. Izmailov \\ Peter the Great St. Petersburg Polytechnic University (SPbPU) \\ 29 Politechnicheskaya str., Saint Petersburg, 195251, Russia
}

Introduction. The article studies the main approaches to systematization of the principles of management of fixed assets of the enterprise. In the introductory part of the article, the importance and necessity of formulating scientifically based principles for managing fixed assets are substantiated. Further, the main part of the article presents the opinions of individual authors regarding the types of principles of management of fixed assets of the enterprise, on the basis of which it is concluded that various authors give their own approaches to the classification of the principles of management of fixed assets in their works. It is proved that none of the above lists of principles is exhaustive, in most of them they are quite general in nature, can be used in relation to not only fixed assets, but almost any object of management at the enterprise.

The results obtained. As a result of the research, a system of special principles for managing the fixed assets of the enterprise was formed, which is presented in the form of a scheme with a detailed description. The system includes the following principles: 1) the principle of integrated accounting of institutions and institutional changes related to fixed assets; 2) the principle of rationalization of transaction costs arising in the process of reproduction of fixed assets; 3) the principle of rational transparency of the process of managing fixed assets; 4) the principle of polysubjectivity of the management of fixed assets of the enterprise; 5) the principle of synergy; 6) the principle of focusing on the long-term maximization of the efficiency and cost of an industrial enterprise. The proposed system of principles for managing fixed assets of an enterprise allows us to fully reflect the specifics of fixed assets as an object of management, as well as to generalize and systematize the principles given in the literature sources under study.

Conclusion. Compliance with the requirements of the proposed principles will ensure high quality of operational and production activities, and also creates conditions for improving the organizational and economic support for maintaining a rational structure of fixed production assets in the process of achieving the goals of economic activity of enterprises and, accordingly, increasing adaptability to market requirements, stimulating the revival of business activity. In turn, an effectively formed system of management of fixed assets will ensure the growth of production volumes, quality improvement, an increase in labor productivity, and a reduction in production costs.

Keywords: fixed assets, management, management principles, fixed assets, efficiency of use, industrial enterprises, system of principles.

For quoting:

Kobzev V. V., Izmailov M. K. System of principles of management of fixed assets // Organizer of production // Organizer of production. 2021. T. 29. № 2. P. 67-76. DOI: 10.36622/VSTU.2021.47.26.007.

\section{Введение}

В современных условиях усиление конкуренции на рынке, изменение структуры спроса, связанное с масштабным использованием новейших цифровых технологий, приводят к необходимости совершенствования экономической политики предприятий, в частности по качественным и количественным характеристикам оборудования, критериям и показателям их использования. Именно поэтому важное значе- 
ние приобретает эффективное управление основными производственными средствами предприятий, что закладывает основы наращивания их конкурентных преимуществ как на внутреннем, так и на внешнем рынках. Исследование состояния и рациональное использование основных фондов на предприятиях является очень актуальным вопросом для предприятий на пути к экономическим реформам, именно благодаря рациональным управленческим решениям можно достичь высокого уровня контроля за обеспеченностью предприятия основными фондами и эффективностью их использования.

Весомый вклад в развитие теоретикометодологических основ управления основными средствами сделали российские ученые. Так, в работах Ю. А. Саблиной [1], В. А. Базовой [2] и Л. М. Мастановой [3] анализируются понятийные аспекты управления основными средствами предприятия, обосновывается важность этого процесса в повышении конкурентоспособности предприятий, улучшении качества выполнения услуг, улучшений условий работы и стабильного увеличения прибыли предприятия. В статьях Л. В. Клочковой [4] и А. В. Мальцева [5] упор делается на выработку рекомендаций, направленных на совершенствование управления основными средствами в целях повышения эффективности их использования. Попытку определить основные принципы управления основными средствами предприятия предпринял Е. А. Дьяченко в своей статье «Принципы и этапы управления основными средствами организации» [6], однако в ней представлен лишь краткий перечень принципов без их подробного обоснования и исследования. Отдельные аспекты построения механизма управления основными средствами, определению этапов и стадий этого процесса исследованы в работах Ю. О. Шавриной, С. С. Таспаева, А. В. Филипповой, А. С. Шибаловой [7], С. С. Меджитова, Е. В. Колесниковой [8], Н. Н. Губернаторовой, Д. Ю. Зубова [9], А. Ю. Зимняковой [10], А. В. Азарова [11] и др.

Проведенный обзор работ указанных авторов показал, что вопросам систематизации принципов управления основными средствами в литературе уделено недостаточно внимания. Всё это указывает на важность и необходимость формулировки научно обоснованных принципов управления основными средствами.
Цель исследования - на основе систематизации мнений отдельных авторов сформулировать и обосновать перечень принципов управления основными средствами.

Для достижения поставленной цели необходимо решить следующие задачи:

- провести исследование мнений отдельных авторов относительно формулировки основных принципов управления основными средствами,

- выявить недостатки предложенных классификаций принципов управления основными средствами,

- предложить собственный вариант принципов управления основными средствами и обосновать возможности его использования в деятельности промышленных предприятий.

Объект исследования - процессы управления основными средствами промышленных предприятий.

Предметом исследования является совокупность теоретических и методических аспектов формулирования принципов управления основными средствами.

Методика исследования. Методологической базой написания статьи является диалектический метод познания, который предполагает рассмотрение всех экономических явлений в динамике и взаимосвязи; теоретические положения, изложенные в работах российских ученых по проблемам обеспеченности, формирования основных средств и эффективности управления ими.

\section{Данные и методы}

При написании статьи были использованы следующие основные методы исследования: индукции и дедукции - для обоснования основных понятий; абстрактно-логический - для теоретического обобщения тенденций и обоснования принципов управления основными средствами; аналогий - для исследования принципов управления основными средствами и связанных с ними понятий и внесение предложений по совершенствованию их классификации; метод аннотирования, реферирования, систематизации и сравнительного анализа - для изучения основных подходов к построению принципов управления осиновыми средствами, монографический - для исследования особенностей применения отдельных принципов управления основными средствами в деятельности промышленных предприятий. 
Методика исследования - на основе систематизации основных видов принципов управления основными средствами выделить недостатки существующих классификаций и предложить собственный перечень принципов с обоснованием их применения в деятельности промышленных предприятий.

\section{Полученные результаты (Results)}

Следует отметить, что различные авторы приводят в своих работах собственные подходы к классификации принципов управления основными средствами.

Так, Э. В. Хлынин и Н. И. Коровкина в своей статье шесть основных принципов управления основными средствами - принципы группировки и классификации, комплексности, системности, аналитичности, сбалансированности, экономической эффективности [12].

По мнению Е. А. Дьяченко перечень принципов управления основными средствами должен включать принцип системности; принцип многовариантности; принцип непрерывности; принцип оптимальности; принцип сбалансированности; принцип гибкости [13].

О. В. Литвинова в работах указывает, что управление основными средствами предприятия должно основываться лишь на трех основных принципах, а именно:

- принцип оптимизации структуры основных средств;

- принцип обеспечения эффективности использования основных средств;

- принцип увеличения стоимости основных средств [14].

Основная цель управления основными фондами предприятия, по мнению А. Е. Тимофеевой и Е. Ю. Левановой, реализуется с учетом следующих принципов:

- принцип качественной и количественной оценки состояния основных средств предприятия;

- принцип раскрытия всех возможных резервов улучшения использования основных средств;

- принцип оценки эффективности основных вариаций модификации и улучшения основных средств;

- принцип раскрытия отклонений, причин и способов устранения ошибок;

- принцип раскрытия слабых мест [15].
В. М. Яценко считает, что для лучшей результативности управленческого механизма на предприятиях нужно учитывать следующие принципы, на которых он базируется.

1. Соответствие (подчиненность) стратегическим целям экономического развития предприятия. Механизм обеспечения эффективного использования основных фондов должен создавать необходимые материальные и финансовые условия для реализации целей и задач экономического развития предприятия в целом.

2. Перспективность. Механизм обеспечения эффективного использования основных средств должен предусмотреть условия для расширения предприятия и повышения эффективности его деятельности в будущих периодах на основе оптимизации соотношения между решением текущих и перспективных задач развития.

3. Обоснованность управленческих решений по характеру воспроизводства основных средств предприятия. Обоснование потребностей в основных средствах следует проводить на основе квалифицированного анализа состояния и использования основных производственных фондов, изучения резервов повышения эффективности их использования.

4. Эффективность. Формирование воспроизводственных процессов на предприятии должно базироваться на условии достижения экономического или социального эффекта, имеющего конечное экономическое измерение.

5. Принцип непрерывности и надежности проявляется в создании таких организационных, технических и хозяйственных условий, при которых может быть достигнут устойчивый и непрерывный процесс производства. Это обеспечивается не только слаженностью и надежностью функционирования управляющей системы и ее элементов, но и объекта управления.

6. Принцип планомерного, пропорционального и динамического управления означает, что система управления должна быть ориентирована не только на текущие цели, но и на долгосрочное развитие компании, и может нести материальную, юридическую и административную ответственность за качество принятого решения и его полное выполнение [16].

Существенно расширил перечень принципов управления основными средствами Н. М. 
Лигай, который в своей диссертации приводит 12 принципов [17]:

- принцип системности определяет процесс формирования основных средств предприятия с целью обеспечения их сбалансированности; соблюдение системности в разработке стратегии и тактики финансирования, а также в реализации запланированных мероприятий через исследование системы взаимосвязанных и взаимосогласованных показателей, например, соотношение основных средств и оборотных активов и т. п.;

- принцип обоснованности обеспечивает формирование основных средств на реалистичных показателях экономического и социального развития предприятия, а также расчетах доходов и расходов деятельности, осуществляемых в соответствии с утвержденными методиками и правилами. Основными такими показателями являются: объемы основных средств, чистая доходность активов, рентабельность, уровень использования ресурсов на 1 руб. чистого дохода, операционные расходы, уровень годности основных средств. Кроме этого, сущность принципа заключается в том, что методы, формы и средства управления должны быть научно обоснованы и проверены на практике, что предполагает непрерывный сбор, обработку и анализ научно-технической, экономической, правовой и других видов информации;

- принцип комплексности включает в себя полный набор функций управления основными средствами - прогнозирование, планирование, контроль, учет, организация, координация, оптимизация;

- принцип адаптивности определяет инновационность формирования основных средств, которая должна быть гибко приспособлена к воздействию внешней и внутренней среды. В соответствии с этим, основным средствам предприятия должен быть характерен инновационный тип развития. Инновацией в этом случае будет выступать использование технологий в сферах, где технологии до этого не применялись или применялись иначе [18];

- принцип целеустремленности ориентирует на цели и задачи, которые на определенный момент ставит перед собой предприятие по формированию и эффективному использованию основных средств через регулирование объемов хозяйствования, ассортимента и качества про- дукции, производительности труда, а также обеспечение соблюдения показателей эффективности хозяйственной деятельности;

- принцип динамичности определяет необходимость систематического привлечения инвестиций для стимулирования формирования и развития основных средств предприятия;

- принцип синергизма (греч. synergos тот, что действует вместе) характеризует дополнительные экономические выгоды, которые возникают при успешном объединении двух или более компаний (слияния или поглощения). Источником этих выгод является более эффективное использование их объединенных основных средств, взаимодополняемость производимых ими технологий и продуктов, возможность сократить расходы на содержание основных фондов и другие аналогичные факторы. В современных условиях лучший результат получают субъекты хозяйствования, которые занимаются хозяйственной деятельностью (создают кластеры), поскольку в таком случае их совокупный имущественный потенциал выше, по сравнению с их единоличным функционированием. Примером реализации принципа синергизма может служить развитие инновационно-активных промышленных кластеров [19];

- принцип оптимальности предполагает, что система управления основными фондами предприятия должна обеспечивать повышение эффективности хозяйственной деятельности с обязательной минимизацией затрат самой системы. Система управления основными средствами не должна создавать большого объема затрат;

- принцип холизма (от греч. holos - целый) [20] основан на идеалистическом учении, в соответствии с которым целое больше, чем сумма его частей. Суть принципа холизма состоит в том, что основные фонды будут формироваться и воспроизводиться эффективно с пользой для предприятия только при условии их синтеза с финансовым, трудовым, природным, ресурсным, информационным, инвестиционно-инновационным и другими видами потенциала предприятия;

- принцип прогнозируемости свидетельствует, что в целях повышения эффективности управления основными средствами, а также снижения уровня затрат и внеплановых потерь руководство предприятия должно постоянно 
проводить мероприятия для прогнозирования возникновения определенных рисков;

- принцип обратной связи реализуется путем проведения диагностики основных средств. Конечные результаты диагностики имущества могут быть использованы для будущего планирования и развития основных средств;

- принцип гибкости предполагает, что система управления основными средствами не является устоявшейся, а изменяется под влиянием внешних и внутренних факторов и, соответственно, до этого основные средства предприятия могут как увеличиваться, так и уменьшаться.

Обобщая изложенные выше подходы, можно сказать, что к настоящему времени, несмотря на некоторые различия в подходах к управлению основными средствами, разработанные принципы управления создают достаточно прочную методологическую основу организации процесса управления для достижения цели предприятия наименее фондозатратным способом.

Вместе с тем, ни один из приведенных перечней принципов управления основными средствами не является исчерпывающим. С каждым новым этапом развития экономики он может дополняться. Не оспаривая значимость приведенных в работах, указанных выше авторов, принципов, необходимо отметить, что они в большинстве носят достаточно общий характер, могут быть использованы в отношении не только основных средств, но практически любого объекта управления на предприятии. Кроме того, в современных условиях главное изменение принципов управления связано с пересмотром роли объектов основных средств в обеспечении успешности бизнеса: в настоящее время результативность использования основных средств, раскрытие их потенциала становятся значимыми факторами достижения целей предприятия и его устойчивой эффективности.

В этой связи сформированы специальные принципы управления основными фондами предприятия, такие как:

1. Принциип комплексного учета институтов и институичональных изменений, связанных с основныли средствами. Содержание предлагаемого принципа означает необходимость обязательного учета институциональных факторов на всех стадиях управления основными фондами предприятия.
В наиболее общем виде институт - это система взаимосвязанных норм неформального и формального характера, а также механизмов принуждения к их исполнению. Институты, связанные с основными средствами, включают в себя не только формальные нормы федерального и регионального законодательства, регламентирующие различные аспекты воспроизводства основных средств (порядка их приобретения, учета, амортизационной политики, эксплуатации, лизинга и т. п.), но и нормы неформальных институтов, например, отраслевых ассоциаций и союзов предпринимателей, так или иначе взаимодействующие с управлением основными средствами. Следует отметить, что многие отечественные промышленные предприятия не в достаточной степени осуществляют учет функционирования формальных и неформальных институтов в процессе управления (вплоть до игнорирования норм специального законодательства об основных фондах), что в конечном итоге снижает в долгосрочной перспективе эффективность управления основными средствами.

2. Принциип рацчионализации трансакциионных издержек, возникающчх в процессе воспроизводства основных средств. Трансакционные издержки представляют собой любые затраты, связанные с обеспечением и защитой прав собственности и обеспечением контрактов. Трансакционные издержки, связанные с основными фондами, включают в себя затраты на осуществление поиска и обработку информации значимой в экономическом аспекте и связанной с приобретением и эксплуатацией основных средств, возможные расходы, обусловленные не вполне корректным выполнением контрактных обязательств поставщиков основных средств, затраты на обеспечение безопасности парка основных средств, возможные коррупционные издержки, связанные с приобретением объектов основных средств. В соответствии с предлагаемым принципом система и методы управления основными средствами предприятия должны обеспечивать рационализацию состава и структуры трансакционных издержек, от чего непосредственно зависят в конечном итоге прибыль, рентабельность и стоимость промышленного предприятия.

3. Принцуип ращуональной транспарентно-
сти прочесса управления основныли


средствами. Принцип означает формирование информационной «прозрачности» системы управления основными средствами, что, в частности, минимизирует риски необоснованного завышения их покупной сметной стоимости (например, посредством внедрения технологии корпоративных тендеров на приобретение основных средств), их нецелевого использования в процессе функционирования и т. п. Вместе с тем, такого рода транспарентность, информационная «прозрачность» управления основными средствами предприятия не может носить абсолютный характер. В частности, подобная транспарентность должна ограничиваться требованиями коммерческой тайны разработок, связанных с основными средствами предприятия.

4. Принщип полисубъектности управления основными средствами предприятия. В соответствии с принципом, круг субъектов управления основными фондами предприятия должен быть достаточно широким и включать в себя следующих основных субъектов:

- непосредственные функциональные и линейные подразделения промышленного предприятия, такие, например, как отдел капитального строительства, отдел технического контроля и др.;

- органы корпоративного управления, в т. ч. совет директоров и общее собрание акционеров предприятия (в части рассмотрения и утверждения долгосрочных программ модернизации основных средств промышленного предприятия);

- рабочие, занятые в процессе эксплуатации основных средств (в части формирования рационализаторских предложений по повышению эффективности их текущего использования в производственном процессе).

5. Принции достижения максимальной синергии путем взаимодействия основных средств предприятия. Принцип предполагает достижение дополнительного положительного эффекта в результате взаимодействия отдельных объектов основных средств, а также взаимодействия основного капитала и иных видов капитала промышленного предприятия.

6. Принщип нащеленности на долгосрочную максимизацию эффективности и стоимости промышленного предприятия. В соответствии с принципом, система и комплекс методов управления основными средствами предприятия должны обеспечивать в долгосрочной перспек- тиве повышение уровня его эффективности и, как следствие, рыночной стоимости. Указанный принцип в определенной степени обобщает содержание выделенных ранее принципов управления основными средствами промышленного предприятия.

По нашему мнению, предлагаемый перечень принципов управления основными средствами промышленного предприятия позволяет наиболее полно отразить особенности управления основными средствами крупных промышленных предприятий, а также обобщить и систематизировать принципы, приведенные в исследуемых литературных источниках. Предложенный перечень принципов, в отличии от рассмотренных в статье принципов управления, позволяет выстроить управление объектами основных средств на промышленном предприятии, обеспечивая ориентацию субъектов управления на повышение эффективности их использования на основе рационализации трансакционных издержек, возникающих в процессе воспроизводства основных средств, рациональной транспарентности, полисубъектности управления. Всё это в конечном итоге способствует долгосрочной максимизации эффективности использования основных средств и повышения стоимости промышленного предприятия в целом.

\section{Заключение}

Проведенное в ходе написания статьи исследование позволило получить следующие результаты:

1) в литературных источниках представлены различные подходы к классификации принципов управления основными средствами предприятия, при этом многие из них перекликаются между собой, некоторые из приведенных принципов, по своему существу, являются правилами поведения руководителей, в полномочия которых входит управление основными средствами.

2) в работе представлен наиболее оптимальный, на наш взгляд, перечень принципов управления основными средствами, включающий шесть основных принципов - принцип комплексного учета институтов и институциональных изменений, связанных с основными средствами, принцип рационализации трансакционных издержек, возникающих в процессе воспроизводства основных средств, принцип рациональной транспарентности процесса 
управления основными средствами, принцип полисубъектности управления основными средствами предприятия, принцип синергизма, принцип нацеленности на долгосрочную максимизацию эффективности и стоимости промышленного предприятия.

3) предложенный перечень принципов основывается на отражении различных сторон отношений управления основными средствами, а также соответствует целям повышения эффективности производственной деятельности промышленного предприятия.

Таким образом, соблюдение требований предлагаемых принципов позволит обеспечить высокое качество оперативно-производственной деятельности, а также создает условия для совершенствования организационноэкономического обеспечения поддержания рациональной структуры основных производственных средств в процессе достижения целей экономической деятельности предприятий и, соответственно, повышение адаптивности к требованиям рынка, стимулирование оживления деловой активности. В свою очередь, эффективный процесс управления основными средствами позволит обеспечить рост объемов производимой продукции, повышение качества, увеличение уровня производительности труда, сокращение затрат на производство.

Дальнейшие исследования в рамках данной тематики будут связаны с попыткой систематизации указанных принципов, а также более подробного изучения их влияния на эффективность производственно-хозяйственной деятельности промышленного предприятия.

\section{Библиографический список}

1. Саблина Ю.А. Управление основными средствами предприятия // Экономика и управление в XXI веке: наука и практика. 2016. № 3. C. $136-139$.

2. Базова В.А. Современное состояние и проблема совершенствавания управления основными средствами //Форум молодых ученых. 2017. № 1 (5). С. 72-75.

3. Мастанова Л.М. Теоретические основы учетно-аналитического обеспечения управления основными средствами организации //Наука и образование сегодня. 2017. № 5 (16). С. 32-34.
4. Крючкова Л.В. Стратегия повышения эффективности использования основных средств на предприятии // Стратегия предприятия в контексте повышения его конкурентоспособности. 2018. № 7. С. 153-156.

5. Мальцев А.В. Совершенствование системы учета и контроля операций с основными средствами //Экономика и социум. 2018. № 1 (44). С. 1207-1211.

6. Дьяченко Е.А. Принципы и этапы управления основными средствами организации // Пути повышения эффективности экономической и социальной деятельности кооперативных организаций. материалы XI международной научно-практической конференции. 2016. С. 197199.

7. Шаврина Ю.О., Таспаев С.С., Филиппова А.В., Шибалова А.С. Управление основными средствами на основе учетно-аналитического обеспечения сельскохозяйственных организаций// Вестник евразийской науки. 2018. Т. 10. № 3. C. 47.

8. Меджитов С.С., Колесникова Е.В. //Политика управления основными средствами организации//Форум молодых ученых. 2019. № 4 (32). С. 715-720.

9. Губернаторова Н.Н., Зубов Д.Ю. Влияние управления основными средствами на обеспечение экономической безопасности организации // Калужский экономический вестник. 2019. № 2. C. 7-15.

10. Зимнякова А.Ю. Анализ эффективности использования основных средств организации //Аллея науки. 2019. Т. 1. № 12 (39). C. $123-127$.

11. Азаров А.В. Комплексный подход к построению системы управления основными средствами предприятия //Теория и практика современной науки. 2017. № 3 (21). С. 29-32.

12. Хлынин Э.В., Коровкина Н.И. Методологические принципы и подходы взаимодействия стратегического и тактического управления основными средствами промышленного предприятия // Известия ТулГУ. Экономические и юридические науки. 2016. №11. С.125-137.

13. Дьяченко Е.А. Принципы и этапы управления основными средствами организации// Пути повышения эффективности экономической и социальной деятельности кооперативных организаций : материалы XI 
международной научно-практической конференции. 2016. С. 197-199

14. Литвинова О.В. Управление и техническая эксплуатация объектов недвижимости, Иркутск: Иркутский национальный исследовательский технический университет, 2015. - 130 с.

15. Тимофеева А.Е., Леванова Е.Ю. Принципы и этапы управления основными средствами организации // Современные проблемы управления финансами компаний и финансовых институтов : сборник материалов Всероссийской заочной научно-практической конференции. 2019. C. $392-395$

16. Яценко В. М. Проблемы эффективного управления основными средствами в газовой отрасли и механизмы их решения (на примере ОАО "Газпром"): дисс. ... д.э.н. / Яценко В.М.М., 2015. - 287 c.

17. Лигай Н.М. Управление устойчивым воспроизводством основных фондов промыш- ленного предприятия: дисс. ... к.э.н.: / Лигай Н. М. СПб., 2019. - 326 с.

18. Темиргалиев Е.Р. Инновационное предпринимательство на рынке доставки товарного бетона Санкт-

Петербурга//Фундаментальные и прикладные исследования в области управления, экономики и торговли : Сборник трудов научной и учебнопрактической конференции. В 3-х частях. 2017. С. $365-3372$

19. Бабкин А.В., Алексеева Н.С. Оценка эффективности функционирования инновационно-активного промышленного кластера // Кластеризация цифровой экономики: глобальные вызовы : Сборник трудов национальной научнопрактической конференции с зарубежным участием. В 2-х томах. Под редакцией Д.Г. Родионова, А.В. Бабкина. 2020. - С. 219-228

20. Большая российская энциклопедия. URL: bigenc.ru

Поступила в редакцию - 06 мая 2021 г. Принята в печать - 16 мая 2021 г.

\section{Bibliography}

1. Sablina Yu. A. Management of fixed assets of the enterprise // Economics and management in the XXI century: science and practice. 2016. No. 3. pp. 136-139.

2. Basova V. A. The current state and the problem of improving the management of fixed assets //Forum of young scientists. 2017. No. 1 (5). pp. 72-75.

3. Mastanova L. M. Theoretical foundations of accounting and analytical support for the management of fixed assets of the organization //Science and education today. 2017. No. 5 (16). pp. 32-34.

4. Kryuchkova L. V. Strategy for improving the efficiency of the use of fixed assets in the enterprise // The strategy of the enterprise in the context of increasing its competitiveness. 2018. No. 7. pp. 153-156.

5. Maltsev A.V. Improving the system of accounting and control of operations with fixed assets //Economy and society. 2018. No. 1 (44). pp. 1207-1211.

6. Dyachenko E. A. Principles and stages of management of fixed assets of the organization / / Ways to improve the efficiency of economic and social activities of cooperative organizations. materials of the XI international scientific and practical conference. 2016. pp. 197-199.

7. Shavrina Yu. O., Taspaev S. S., Filippova A.V., Shibalova A. S. Management of fixed assets on the basis of accounting and analytical support of agricultural organizations// Bulletin of Eurasian Science. 2018. Vol. 10. No. 3. p. 47.

8. Medzhitov S. S., Kolesnikova E. V. //Management policy of the organization's fixed assets//Forum of young scientists. 2019. No. 4 (32). pp. 715-720.

9. Gubernatorova N. N., Zubov D. Yu. The influence of management of fixed assets on ensuring the economic security of an organization / / Kaluga Economic Bulletin. 2019. No. 2. pp. 7-15.

10. Zimnyakova A. Yu. Analysis of the effectiveness of the use of fixed assets of the organization //Science Alley. 2019. Vol. 1. No. 12 (39). pp. 123-127.

11. Azarov A.V. An integrated approach to building a management system for fixed assets of an enterprise //Theory and practice of modern science. 2017. No. 3 (21). pp. 29-32.

12. Khlynin E. V., Korovkina N. I. Methodological principles and approaches of interaction of strategic and tactical management of fixed assets of an industrial enterprise // News of TulSU. Economic and legal sciences. 2016. No. 1-1. pp. 125-137. 
13. Dyachenko E. A. Principles and stages of management of fixed assets of the organization// Ways to improve the efficiency of economic and social activities of cooperative organizations: materials of the XI International scientific and practical conference. 2016. pp. 197-199

14. Litvinova O. V. Management and technical operation of real estate objects, Irkutsk: Irkutsk National Research Technical University, 2015. - 130 p.

15. Timofeeva A. E., Levanova E. Yu. Principles and stages of management of fixed assets of the organization / / Modern problems of financial management of companies and financial institutions : a collection of materials of the All-Russian correspondence scientific and practical conference. 2019. pp. 392395

16. Yatsenko V. M. Problems of effective management of fixed assets in the gas industry and mechanisms for their solution (on the example of Gazprom): diss. ... Doctor of Economics / Yatsenko V. M.-M., 2015. - $287 \mathrm{p}$.

17. Ligay N. M. Management of sustainable reproduction of fixed assets of an industrial enterprise: diss. ... Candidate of Economics: / Ligay N. M. St. Petersburg, 2019. - 326 p.

18. Temirgaliev E. R. Innovative entrepreneurship in the market of delivery of ready-mixed concrete in St. Petersburg//Fundamental and applied research in the field of management, economics and trade: Proceedings of the scientific and educational-practical conference. In 3 parts. 2017 - - pp. 365-3372

19. Babkin A.V., Alekseeva N. S. Evaluation of the effectiveness of the functioning of an innovative and active industrial cluster / / Clustering of the digital economy: global challenges : Proceedings of the national scientific and practical conference with foreign participation. In 2 volumes. Edited by D. G. Rodionov, A.V. Babkin. 2020. - pp. 219-228

20. The Great Russian Encyclopedia. URL: bigenc.ru

Received - 06 May 2021

Accepted for publication - 16 May 2021 